\title{
Reconfiguration-Assisted Charging in Large-Scale Lithium-ion Battery Systems
}

\author{
Liang He ${ }^{1}$, Linghe Kong ${ }^{1}$, Siyu Linn ${ }^{1}$, Shaodong Ying ${ }^{1}$, Yu Gu${ }^{1}$, Tian He${ }^{2}$, Cong Liu ${ }^{3}$ \\ ${ }^{1}$ Singapore University of Technology and Design, Singapore \\ ${ }^{2}$ University of Minnesota, Minneapolis, MN, USA \\ ${ }^{3}$ University of Texas at Dallas, Dallas, TX, USA
}

\begin{abstract}
Large-scale Lithium-ion batteries are widely adopted in many systems such as electric vehicles and energy backup in power grids. Due to factors such as manufacturing difference and heterogeneous discharging conditions, cells in the battery system may have different statuses such as diverse voltage levels. This cell diversity is commonly known as the cell unbalance issue, which becomes more critical as the system scale increases. The cell unbalance issue not only significantly degrades the system performance in many aspects, but may also cause system safety issues such as the burning of battery cells and thus increase system vulnerability. In this paper, based on the advancement in reconfigurable battery systems, we demonstrate how to utilize system reconfiguration flexibility for achieving an efficient charging process for the battery system. With the proposed reconfiguration-assisted charging, the cells in the system are categorized according to their voltages, and the charging process is evolutionarily carried out in a category-by-category manner. For the charging of cells in a given category, a graph-based algorithm is presented to obtain the desired system configuration. We extensively evaluate the reconfiguration-assisted charging through small-scale implementation and large-scale trace-driven simulations. The results demonstrate that our proposed techniques can achieve a $25 \%$ increase on average on charged capacities of individual cells while yielding a dramatically reduced variance.
\end{abstract}

\section{INTRODUCTION}

Large-scale battery systems consisting of hundreds or thousands of battery cells are widely adopted in elec-

Permission to make digital or hard copies of all or part of this work for personal or classroom use is granted without fee provided that copies are not made or distributed for profit or commercial advantage and that copies bear this notice and the full citation on the first page. To copy otherwise, to republish, to post on servers or to redistribute to lists, requires prior specific permission and/or a fee.

ICCPS'14, April 14-17, 2014, Berlin, Germany. Copyright 2013 ACM 978-1-4503-1996-6/13/04 . . \$15.00. tric vehicles 19, 22, 23, 25, 40, residential power backup systems [3, airplane electrical systems [1,5], etc. While such large-scale systems are able to provide powerful energy supply, it also introduces new challenges, among which, the cell unbalance issue in the system is one of the most critical problems [28, 31, 32.

Ideally, cells in a battery system are desired to have similar capacity and characteristics 21. However, due to various reasons such as manufacturing difference, cell aging, operational conditions, and chemical property variations, the cells' characteristics demonstrate significant dynamics during the operating cycles over an extended time 34. The cell unbalance issue becomes even more severe for large-scale battery systems, because many such systems can simultaneously support multiple loads with different energy requirements 24. This implies that cells in the system may have very different discharge profiles.

In battery systems with unbalanced cells, to avoid the cell overheating and the thermal runaway issue, the system operation is fundamentally limited by the weakest cell [21,33]. This is especially critical in a serial configuration of cells which is only as strong as the weakest link. As a result, the imbalance among a set series connected cells, which is commonly (but not only) reflected by the difference among their voltages, prevents them to supply their capacities fully or being charged fully, and consequently degrades the cell operation time, state of health, life cycles [7,36]. The cell unbalance issue may even cause severe system safety issues such as the burning of battery cells [5].

Exploring the system reconfiguration flexibility is a new dimension to improve the battery system performance [11,28, in which the system can adaptively adjust the cells configuration (e.g., connected in series, in parallel, or in a hybrid manner) based on the real-time system conditions such as cell voltages [37, cell temperature [31, and load requirements 28]. Many existing investigations focus on the discharging scenario of the battery system with the objective of maximizing the system energy utilization efficiency [24,32. Com- 
plementary to these existing works, in this paper we explore how the reconfiguration flexibility can assist the charging scenario of the widely utilized Lithium-ion battery systems. Different from the system discharging scenario where the discharging current of individual cells is desired to be small due to the rate-capacity effect of battery cells 29], the desired current when charging battery cells is determined by the cell's voltage level [21. Furthermore, with a fixed charging voltage as in many off-the-shelf battery chargers [8], the cell voltage also directly affects the actual charging current, as we will see in Section 3 .

Our basic idea in the design of the reconfigurationassisted charging is the following: by adjusting the system configuration, we can control the charging currents for individual cells in the system to match them with the desired current levels, so that the cells in the system can be efficiently charged to their maximal capacity. Our main contributions in this work include:

- To the best of our knowledge, our work is the first attempt to explore the system reconfiguration flexibility to achieve the optimal charging process in large-scale battery systems.

- Observing the facts that cells with different voltages may desire different charging currents and that only cells with similar voltages should be connected in series, we propose a novel cell categorization method based on their individual voltages and the system hardware constraints, with which the cells with similar voltages (and thus desiring similar charging currents) are organized into the same category. Then the system charging is evolutionarily carried out in a category-by-category manner.

- For the charging of cells in a given category, we transform the problem of identifying the optimal system configuration for the charging process to a path selection problem in the abstracted cell graph. We prove the problem is NP-hard, and propose an algorithm to obtain the near-optimal system configuration. Moreover, to facilitate our control over the charging currents of cells at finer granularity, a set of adjustable resistor arrays is introduced into the design while the additional energy loss on them is minimized.

- We evaluate the proposed reconfiguration-assisted charging process through both small-scale experiments and large-scale trace-driven simulations. The results show the reconfiguration-assisted charging process can improve the average charged capacities of individual cells by about $25 \%$ and dramatically reduce their variance among cells.

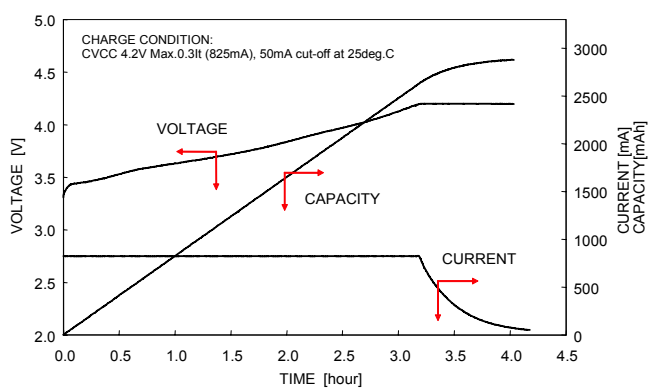

Figure 1: The desired charging process for the Panasonic NCR18650 Lithium-ion battery [11].

The paper is organized as follows. The preliminaries is presented in Section 2, The basic principle of our design is introduced in in Section 3. The proposed reconfiguration-assisted charging process is presented in Section 4 and Section 5. Our experiment and simulation are presented in Section 6 and Section 17, respectively. Section 8 reviews the literature, and Section 9 concludes the paper.

\section{PRELIMINARIES}

\subsection{Problem Statement}

The cell unbalance issue makes the efficient charging of the large-scale battery systems challenging. This is due to the facts that the charging of series connected cells has to be terminated when any of the cells reaches its voltage upper boundary 31 and cells with different voltage levels may desire different charging currents [21. As an example, the desired charging process of the Panasonic NCR18650 Lithium-ion battery cell [10] is shown in Fig. 1. We can see that during the early phase of the charging process, the cell is desired to be charged with a relatively large constant current (e.g., about $0.825 \mathrm{~A}$ ). This is referred to as the Constant Current Charging (CC-Chg) phase. Then when the cell voltage reaches a certain level (e.g., about 4.19 volt), the charging process is changed to a Constant Voltage Charging (CV-Chg) phase, during which the cell is charged with a constant voltage and the charging current decreases to a small value (e.g., about $50 \mathrm{~mA}$ ) till the cell is fully charged (e.g., the cell voltage reaches about 4.20 volts). Note although the specific current and voltage voltages (e.g., $0.825 \mathrm{~A}$ and $4.19 \mathrm{~V}$ ) are only for the NCR18650 Lithium-ion battery cells, the CCChg and CV-Chg phases are shared by all Lithium-ion batteries. The detailed explanation on the charging process of Lithium-ion cells can be found in [21.

This voltage-dependent charging currents indicate that with the cell unbalance issue, individual cells in the system may require different charging currents. However, most off-the-shelf multi-cell chargers treat the cells 
identically, e.g., the LP2952-based 3-cell charger from Texas Instruments always charges the cells with the same current 8. Clearly, this homogeneous charging current causes deviation between the actual charging currents and the desired charging currents for individual cells.

A charging current different from the desired level significantly degrades the charging process 29. When the charging current is too large, not all the provided energy can be effectively accepted by the cell, and thus reduces the energy efficiency of the charging process. Furthermore, an over-large charging current also easily leads to the cell overheating and thus the thermal runaway issue 21. The thermal runaway issue is especially critical for Lithium-ion battery cells because of their high energy density, which have caused serious accidents, e.g., the Boeing 787 Dreamliner battery incidents [5]. On the other hand, a too small charging current unnecessarily prolongs the charging time [39], increases the cell internal resistance [35, and even prevents the cell from being charged 7 .

In this paper, we tackle the efficient charging of largescale Lithium-ion battery systems by exploring the system reconfiguration flexibility. With the offered reconfiguration flexibility, we can organize the cells with similar voltages together, and then charge them with their desired charging current. However, as the configuration flexibility offered by the system is normally constrained, we need to identify the optimal system configuration to achieve a high charging performance, and adaptively adjust it according to the real-time cell voltages.

\subsection{System Model}

The system model overview for the reconfigurationassisted charging process is presented in Fig. 2] in which the charger imposes a constant DC charging voltage $V$ to the $N$-cell reconfigurable battery pack 1 , and the cell voltages can be monitored by the voltage sensors equipped on them. Note that many research efforts have been devoted to achieve high system reconfiguration flexibility with low system complexity [13]26] (e.g., Figure 3 shows our implementation of a 4-cell reconfigurable testbed based on the circuit design proposed in 31), and our aim in this work is to demonstrate how this offered reconfiguration flexibility can be utilized to improve the system charging process.

The system determines its optimal configuration to accept the charging voltage (and power) to achieve the optimal charging processes for individual cells. Note that for many existing battery systems, the charging

\footnotetext{
${ }^{1}$ Here we assume the charger power is high enough for the charging process under consideration. This is feasible in practice because the capable charger is always selected according to the corresponding battery system.
}

terminals can be directed connected to any cells through the backbone buses 33 . In the following, we assume this full connectivity of the charging terminals.

To facilitate the charging current control at finer granularity, we introduce a set of adjustable resistor arrays (with unit resistor $r_{0}$ ) into the system, as shown in Fig. 4. The relays equipped on the resistors allow us to control the number of resistors included in the charging process and thus control the charging current. Clearly, an additional questions with regard to these resistor arrays is how much energy will be lost on them and how to minimize it, which we will elaborate in Section 5

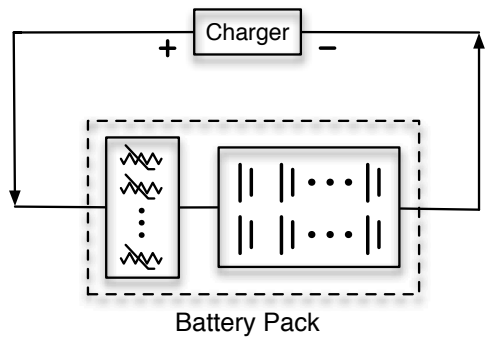

Figure 2: Overview of the system model.

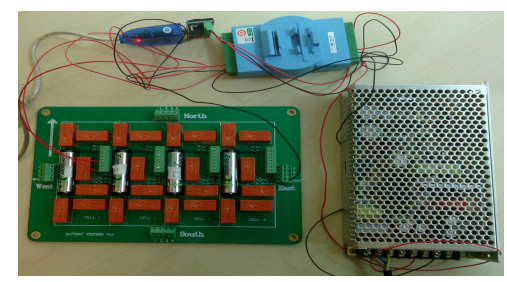

Figure 3: Our 4-cell reconfigurable testbed.

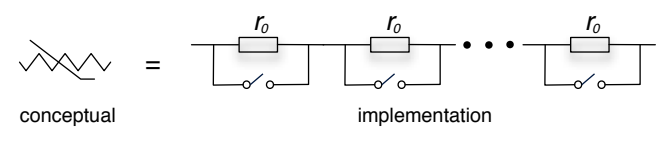

Figure 4: Design of the resistor arrays.

\subsection{Graph Representation}

As proposed in our previous work 24, the cells in the system can be represented by a weighted directed graph $\mathcal{G}=\{\mathcal{V}, \mathcal{E}, \mathcal{W}\}$, where 1 ) each vertex in $\mathcal{V}$ represents a cell in the system, and thus $|\mathcal{V}|=N$;2) $\mathcal{E}$ reflects how these cells can be connected to each other and thus captures the system configuration flexibility: an edge $v_{i} \rightarrow v_{j} \in \mathcal{E}$ if and only if the current can flow from $v_{i}$ to $v_{j}$ without passing any other cells; 3 ) the weight set $\mathcal{W}$ on the vertices captures the cell voltages. 


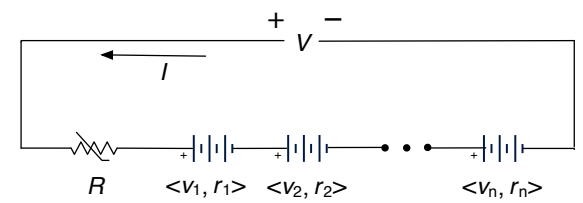

Figure 5: Charging cells connected in series.

This graph representation facilitates our design by bridging the gap between the desired system configuration for the charging process and the advancements in graph theory, as will be seen in Section 5. Before describe our design on the reconfiguration-assisted charging process in detail, in the next section, we first investigate how to control the charging current of individual cells by adjusting the system configuration and the amount of adopted resistors.

\section{DESIGN PRINCIPLE}

For a set of cells connected in series (i.e., a cell string), the voltage they provide is the sum of their individual voltages and each cell has the same discharging/charging current 4. This simple fact motivates us the design principle of the proposed charging algorithm.

Let us consider the example shown in Fig. 囵, If certain subset of $n$ cells in the system are connected in series along with a resistor $R$, then with a charging voltage $V$, the charging current for these cells can be calculated as

$$
I=\frac{V-\sum_{1}^{n} v_{i}}{\sum_{1}^{n} r_{i}+R},
$$

where $\left\langle v_{i}, r_{i}\right\rangle$ is the voltage and internal resistance of the $i$ th cell, and $\left(V-\sum_{1}^{n} v_{i}\right)$ is the effective charging voltage imposed on the cell string. Clearly, the condition that $V>\sum_{1}^{n} v_{i}$ must be satisfied to charge the cells.

For many types of battery cells, their internal resistances increase as the cells are being discharged 29, 39 . For the charging process, this indicates a smaller $r_{i}$ as $v_{i}$ increases. However, for Lithium-ion battery cells widely adopted in large battery systems such as electric vehicles and airplanes [1,30, the internal resistance is relatively stable during the charging/discharging process [7. Fig. 6] shows our measurement results on the internal resistance of a Panasonic NCR18650 Lithiumion battery cell during the charging process. We can see that the resistance is stable (about $0.06 \mathrm{ohm}$ ) throughout the charging process. As a result, we simplify the presentation by assuming a stable cell internal resistance (denoted as $r$ ) during the charging process in the rest of the paper. However, this simplification is not required in our design, as the real-time cell resistance can be easily measured (as shown in Fig. 6) and adopted in Eq. (1).

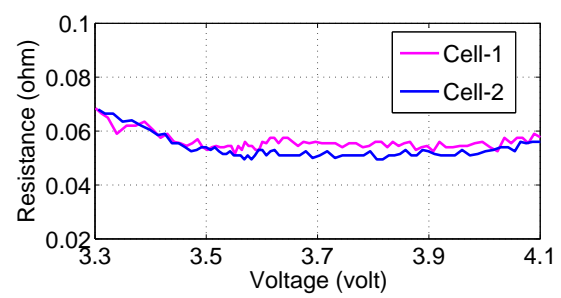

Figure 6: Resistance of NCR18650 cell.

From Eq. (11), we can see that the charging current of these series connected cells can be controlled by jointly considering

- how many (and which) cells should be adopted to compose the string (i.e., controlling $\sum_{1}^{n} v_{i}$ and $\left.\sum_{1}^{n} r_{i}\right)$

- the amount of additional resistance connected along the string (i.e., controlling $R$ ).

Motivated by these observations, we first identify the optimal system configuration for the charging process (i.e., identifying how the cell strings should be formed), and then involve a proper amount of resistors to achieve the optimal charging process based on the system configuration.

The proposed reconfiguration-assisted charging algorithm consists of two steps. We first categorize the cells according to their voltage levels, and then with the assistance of system reconfiguration, the charging process is carried out in a category-by-category manner. In the next two sections, we introduce the two steps in detail respectively.

\section{VOLTAGE-BASED CLASSIFICATION}

As mentioned in Section 3, the cells involved in the same string have the same charging current, and thus it is intuitive to compose a cell string only with cells of similar voltages (and thus desire similar charging currents). This also agrees with the fact that connecting cells with quite different voltages in series is not desired in battery systems, which makes the cell unbalance issue even more critical 4,12. In the first step of our design, we categorize the cells with similar voltages into the same category, based on which the charging process is performed afterwards.

\subsection{Basic Classification Idea}

We discretize the range of cell voltages into a set of voltage intervals and categorize cells accordingly. Specifically, the range of all possible cell voltages is divided into $M$ intervals

$$
\left\{\left[v_{c}^{0}, v_{c}^{1}\right),\left[v_{c}^{1}, v_{c}^{2}\right), \cdots,\left[v_{c}^{M-1}, v_{c}^{M}\right]\right\},
$$


where $v_{c}^{0}=v_{\text {cutoff }}$ (i.e., the voltage defines the empty state of the cell) and $v_{c}^{M}=v_{\text {full }}$ (i.e., the fully charged cell voltage). For example, for most Lithium-ion battery cells, $v_{\text {cutoff }} \approx 3.20$ volt and $v_{\text {full }} \approx 4.20$ volt. Then a total number of $M$ cell categories are formed according to these voltage intervals. Denote the voltages of individual cells as $\left\{v_{1}, v_{2}, \cdots, v_{N}\right\}$, then cell $i$ is classified into the $j$ th category if and only if $v_{i} \in\left[v_{c}^{j-1}, v_{c}^{j}\right)$. For the ease of description, we refer the interval (category) with voltages $\left[v_{c}^{j-1}, v_{c}^{j}\right)$ as the $j$ th voltage interval (category).

After this classification, cells in the same category have similar voltage levels and desire similar charging currents. We use the mean of the voltages of these cells to approximate the voltages of cells in the $j$ th category, denoted as $\hat{v}_{c}^{j}$, and use the corresponding desired charging current at $\hat{v}_{c}^{j}$ as their desired charging current.

Clearly, with the proposed approximation, the more voltage intervals we discretize, the higher the accuracy in achieving the desired charging current. However, due to the constraints of hardware components such as the unit resistor $r_{0}$ and the voltage sensor on each cell, an excessively high discretization degree may not be necessary. Next we explain how to discretize the voltage range with given hardware constraints for the CC-Chg and CV-Chg phases, respectively.

\subsection{Discretization during CC-Chg Phase}

The unit resistor $r_{0}$ determines the minimum voltage changes we can differentiate by adjusting the additionally included resistors. To show this clearly, let us consider an $x$-cell string with cell voltage $\hat{v}$. We will calculate the smallest $\hat{v}^{\prime}\left(\hat{v}^{\prime}>\hat{v}\right)$ that the system can accurately differentiate from $\hat{v}$ with a given $r_{0}$. From Eq. (1), we know

$$
\left\{\begin{array}{l}
\hat{v}=\frac{V-x \cdot r \cdot \hat{I}-y \cdot r_{0} \cdot \hat{I}}{x} \\
\hat{v}^{\prime}=\frac{V-x \cdot r \cdot \hat{I}-y^{\prime} \cdot r_{0} \cdot \hat{I}}{x}
\end{array}\right.
$$

where $\hat{I}$ is the desired charging current, which is constant during the CC-Chg phase, and $y$ and $y^{\prime}$ are the numbers of unit resistors involved in the cell string when the cell voltages are $\hat{v}$ and $\hat{v}^{\prime}$, respectively. Since $\hat{v}^{\prime}>\hat{v}$, it is clear that $y^{\prime}<y$. From Eq. (2), we can see that

$$
\hat{v}^{\prime}-\hat{v}=\frac{\left(y-y^{\prime}\right) \cdot r_{0} \cdot \hat{I}}{x} .
$$

Because $y, y^{\prime}$, and $x$ can only take positive integer values (i.e., $y, y^{\prime}, x \in Z^{+}$), the minimal voltage increase is achieved when 1) $y-y^{\prime}=1$, and 2) $x$ reaches the maximal number of cells that can be connected in series in a feasible cell string. We refer a cell string to be feasible for the charging process if this string can be charged with a voltage $V$. From Eq. (1), we know

$$
\begin{aligned}
x_{\max }= & \arg \max _{x}\left\{\frac{V-x \cdot \hat{v}}{x \cdot r+y \cdot r_{0}}=\hat{I}\right\} \\
\text { s.t. } \quad & \left\{r, r_{0}, \hat{v}, V, \hat{I}\right\}>0, \\
& \{x, y\} \in Z^{+} .
\end{aligned}
$$

From Eq. (3) and (4), we can see that by adjusting the additionally included resistors, the smallest voltage increase that a system can differentiate from $\hat{v}$ is

$$
\hat{v}^{\prime}=\hat{v}+\frac{r_{0} \cdot \hat{I}}{x_{\max }} .
$$

As a result, starting from the cutoff voltage $v_{c}^{0}=$ $v_{\text {cutoff }}$, we iteratively discretize the voltage intervals during the CC-Chg phase according to Eq. (5), and achieves the highest accuracy the adopted hardware can provide.

\section{Example.}

Let us consider the charging chart of the NCR18650 battery shown in Fig. 1 as an example, where $v_{\text {cutoff }} \approx 3.30$ volt and the maximal voltage $v_{\mathrm{cc}-\max } \approx 4.19$ volt for the CC-Chg phase. When a unit resistor of $1 \mathrm{ohm}$ is adopted, the battery voltage during the $\mathrm{CC}$-Chg phase is divided into 8 intervals (i.e., $[3.300,3.414), \quad[3.414,3.517), \quad[3.517,3.620)$, $[3.620,3.723), \quad[3.723,3.841), \quad[3.841,3.959)$, $[3.959,4.077), \quad[4.077,4.190))$, and thus 8 corresponding cell categories are formed. The number of voltage intervals (and thus the cell categories) is reduced to 4 when the unit resistance increases to $2 \mathrm{ohm}$ (i.e., $[3.30,3.517),[3.517,3.723),[3.723,3.959)$, $[3.959,4.190))$.

\subsection{Discretization during CV-Chg Phase}

Different from the CC-Chg phase where the cell voltage increases relatively fast, during the CV-Chg phase, the increase of the cell voltage is slow. For example, for the NCR18950 battery cell shown in Fig. 1, the CVChg phase starts when the cell voltage reaches about 4.19 volt, and the entire charging process terminates when the cell voltage reaches about 4.20 volt, indicating a total voltage increase of only 0.01 volt during the CV-Chg phase lasting about 1 hour. On the other hand, the desired charging current decreases dramatically during this phase, indicating that a relatively large number of voltage intervals are needed to achieve a good matching with the desired currents. Therefore, we discretize the voltage intervals during the $\mathrm{CV}$-Chg phase based on the highest measurement accuracy of the adopted voltage sensor, e.g., 0.002 volt in our testbed shown in Fig. 3 . 


\section{CHARGING FOR EACH CATEGORY}

After the cell categorization, the reconfigurationassisted charging process is evolutionarily carried out in a category-by-category manner with the ascending order of their corresponding voltages. Specifically, the cells in the 1st category are charged first until their voltages evolve into the 2nd interval, and thus these category-1 cells becomes category- 2 cells. Then the cells in the 2 nd category are charged until their voltages increase to the 3rd interval. This process continues until the all the cells in the system are fully charged.

With the proposed classification method, cells in the same category have similar voltage levels and desire similar charging currents, which facilitates us to achieve the optimal charging process by connecting them in series. Next we describe how to identify the desired configuration to perform the charging process for the cells in a given category.

\subsection{Graph-based Problem Transformation}

Let us consider the case where we are trying to identify the configuration for the $N_{j}$ cells in the $j$ th category, each with an approximated voltage $\hat{v}_{c}^{j}$. Denote the cells in the system as $\left\{B_{1}, B_{2}, \cdots, B_{N}\right\}$, and the cell categories as $\left\{C_{1}, C_{2}, \cdots, C_{M}\right\}$.

To charge these $N_{j}$ cells in the $j$ th category with the desired charging current, our approach is to identify a desired series configuration of these cells, and then by controlling the additional resistance along the string, these cells can be optimally charged. In practice, It is very likely that not all of these cells can be charged with a single cell string, because of either the limitation of their configuration flexibilities (i.e., not all these $N_{j}$ cells can be connected in series) or the requirement on a feasible string (i.e., the voltage sum of these cells is too high for them to be charged in series with voltage $V$ ). As a result, we may need to identify multiple cell strings, as well as the proper amount of additional resistance for each string, and then connect them in parallel to perform the charging 2 Since adding additional resistance to the cell strings introduces additional energy loss, we aim to minimize the amount of resistance involved in these identified cell strings.

As mentioned in Section 2 for the $j$ th category, its $N_{j}$ cells and their configuration flexibilities can be represented by a directed graph $\mathcal{G}_{j}=<\mathcal{V}_{j}, \mathcal{E}_{j}, \mathcal{W}_{j}>$, which is a subgraph for the $N$-cell system: $\mathcal{G}_{j} \subseteq \mathcal{G}$. With this graph representation, any cell string in the battery system can be captured by a simple path in the graph. Thus our objective in identifying cell strings can be transformed to identify a set of paths such that

\footnotetext{
${ }^{2}$ Note the feasibility of charging these cell strings in parallel can be guaranteed with the fully connected charging terminals.
}

- each vertex in the graph is involved in one and only one path, indicating the paths are disjoint and cover the vertex set $\mathcal{V}_{j}$ (and thus it is possible to achieve the desired charging current for all the cells);

- each path involves no more than $x_{\max }$ vertices (and thus the cell string can be charged with voltage $V$ ).

Denote $z$ as the number of identified disjoint paths, i.e., $P a t h_{1}^{j}$, Path $_{2}^{j}, \cdots$, Path $_{z}^{j}$. Denote $x_{k}$ and $y_{k}(k=$ $1,2, \cdots, z)$ as the number of vertices in $P_{a t h}^{j}$ and the number of unit resistors included along Path $_{k}^{j}$, respectively. It is clear that $\sum_{k=1}^{z} x_{i}=N_{j}$. For each cell $B_{i}$ and $P a t h_{k}^{j}$, we define indicator variable

$$
b_{i k}^{j}= \begin{cases}1 & \text { if } B_{i} \in C_{j} \text { and } B_{i} \in \text { Path }_{k}^{j} . \\ 0 & \text { otherwise }\end{cases}
$$

Incorporating the objective in minimizing the added additional resistors, our problem can be mathematically formulated as

$$
\begin{array}{ll} 
& \min \sum_{k=1}^{z} y_{k} \cdot r_{0} \\
\text { s.t. } \quad \forall k, \frac{V-x_{k} \cdot \hat{v}_{c}^{j}}{x_{k} \cdot r+y_{k} \cdot r_{0}}=\hat{I}_{j} \\
& \forall i, \sum_{k=1}^{z} b_{i k}^{j}=1 . \\
& \forall k, 1 \leq x_{k} \leq x_{\max } .
\end{array}
$$

where $\hat{I}_{j}$ is the desired charging current. The first constraint guarantees the desired charging current on each cell string, and the second constraint guarantees each cell is included in only one identified cell string. With rearrangements, we have

$$
\begin{aligned}
\min \sum_{k=1}^{z} y_{k} \cdot r_{0} & \Leftrightarrow \min \sum_{k=1}^{z} \frac{V-x_{k} \cdot \hat{v}_{c}^{j}-x_{k} \cdot r \cdot \hat{I}_{j}}{\hat{I}_{j}} \\
& \Leftrightarrow \min \left\{z \cdot V-\hat{v}_{c}^{j} \cdot N_{j}-r \cdot \hat{I}_{j} \cdot N_{j}\right\} \\
& \Leftrightarrow \min z .
\end{aligned}
$$

As a result, the objective of minimizing the additional resistance can be transformed to minimize the number of cell strings that involve each cell only once. This is similar to the Minimum Path Cover (MPC) problem [42,43, with the additional requirement that $x_{k} \leq x_{\max }$.

THEOREM 1. The problem of finding the minimum number of cell strings that 1) conforming to the constraint on the maximal number of cells involved in each string, and 2) involving each cell once and only once, is NP-hard. 
This theorem can be easily proved by contradiction: if a polynomial time algorithm exists for our problem, the MPC problem can be solved in polynomial time as well. This contradicts with the NP-hard property of the MPC problem [43].

\subsection{Algorithm Design}

\subsubsection{Observation}

The MPC problem not only helps us to show that identifying the optimal charging configuration is NPhard, but also inspires us to design a near optimal solution. The key observation is the following: for $d i$ rected acyclic graphs, the MPC problem can be solved in polynomial time. For example, Dilworth et al. have shown that by duplicating the vertex set of the given directed acyclic graph, the MPC problem can be transformed to a maximum matching problem [20], which can be solved by the famous Hopcroft-Karp algorithm with $\mathcal{O}(\sqrt{\mathcal{V}} \mathcal{E})[27$.

Observing the existence of polynomial time algorithms for this special case, our approach therefore is to prune $\mathcal{G}_{j}$ such that we can leverage the existing algorithms to identify the desired system configuration. The pruning of $\mathcal{G}_{j}$ needs to achieve two goals: first, we need to prune $\mathcal{G}_{j}$ to be acyclic, and second, we need to guarantee the obtained paths involve no more than $x_{\max }$ vertices.

\subsubsection{Pruning the Graph}

In our design, we achieve the above mentioned objectives by identifying and breaking all paths involving $\left(x_{\max }+1\right)$ vertices in $\mathcal{G}_{j}$. For the ease of description, denote the resultant graph after pruning as $\mathcal{G}_{j}^{\prime}$. Then we show that $\mathcal{G}_{j}^{\prime}$ is acyclic and does not contain any path that involves more than $x_{\max }$ vertices. Therefore, we can apply existing algorithms to $\mathcal{G}_{j}^{\prime}$ to obtain the desired battery configuration.

Denote $\mathbb{A}=\left\{\alpha_{i j}\right\}\left(i, j=1,2, \cdots, N_{j}\right)$ as the adjacent matrix of $\mathcal{G}_{j}$, i.e., $\alpha_{i j}=1$ if there is an edge from vertex $i$ to vertex $j$ in $\mathcal{E}_{j}$, and $\alpha_{i j}=0$ otherwise. Such adjacent matrix has the following important property.

$\underline{\text { PROPERTY }}$ 1. For any given graph $\mathcal{G}$, the element $\alpha_{i j}^{k}$ in the $k$-th power of its adjacent matrix (i.e., $\mathbb{A}^{k}$ ) is the number of paths from vertex $i$ to $j$ in $\mathcal{G}$ involving $(k+1)$ vertices.

This property holds for general graphs no matter whether it is cyclic or acyclic [42].

Thus if we multiply $\mathbb{A}$ with itself for $\left(x_{\max }-1\right)$ times (i.e, $\left.\mathbb{A}^{x_{\max }}\right)$, then the value of $\alpha_{i j}^{x_{\max }}$ indicates the number of paths involving $\left(x_{\max }+1\right)$ vertices from vertex $i$ to $j$. The time complexity for this matrix multiplication is $\mathcal{O}\left(x_{\max } \cdot N_{j}^{2.37}\right)[18$.
Clearly, for any vertex pair $(i, j)$ with $\alpha_{i j}^{x_{\max }}>0$, we can identify these $\left(x_{\max }+1\right)$-vertex paths by checking the neighbours of $i$ and $\mathbb{A}\left(x_{\max }-1\right)$ in a recursive manner. The time complexity to identify all these $\left(x_{\max }+1\right)$ vertex paths from $i$ to $j$ is $\mathcal{O}\left(d^{x_{\max }}\right)$, where $d$ is the outdegree of vertex $i$. As a result, a time of $\mathcal{O}\left(d^{x_{\max }} \cdot N_{j}^{2}\right)$ is needed to identify all the $\left(x_{\max }+1\right)$-vertex paths in $\mathcal{G}_{j}$. Note that for battery systems, the vertex out-degree $d$ will not be large due to the consideration of system implementation complexity [6, 24].

As an example, let us consider the battery subgraph (i.e., $\mathcal{G}_{j}$ ) shown in Fig. 7(a), and assume any feasible cell string can involve at most $x_{\max }=3$ cells. The adjacent matrix $\mathbb{A}$ for the graph is shown in Fig. 7(b). Since each feasible path can involve at most 3 vertices, we calculate $\mathbb{A}^{3}$ as shown in Fig. [7(c). Because $\alpha_{1,6}^{3}=1$, we know there is one 4 -vertex path from vertex 1 to vertex 6 (i.e., $1 \rightarrow 2 \rightarrow 3 \rightarrow 6$ as shown in Fig. 7(a)). Similarly, because $\alpha_{2,5}^{3}=1$ and $\alpha_{4,5}^{3}=1$, we know another two 4 -vertex paths exists from vertex 2 to vertex 5 and from vertex 4 to vertex 5 respectively.

After identifying these $\left(x_{\max }+1\right)$-vertex paths, by removing at most one edge from each of them, we can break each of these paths into two shorter paths each involving at most $x_{\max }$ vertices (note it is possible for these shorter paths to involve only a single vertex). The pruned graph after breaking these $\left(x_{\max }+1\right)$-vertex paths is denoted as $\mathcal{G}_{j}^{\prime}$.

As we will show shortly, we want to minimize the number of removed edges when breaking the $\left(x_{\max }+1\right)$ vertex paths. To achieve this, for each edge $e_{i} \in \mathcal{E}_{j}$, we use the following indicator variable to describe whether it is involved in the $k$ th path

$$
g_{i k}=\left\{\begin{array}{ll}
1 & \text { if } e_{i} \text { is on } \text { Path }_{k} \\
0 & \text { otherwise }
\end{array},\right.
$$

and further define another indicator to denote whether edge $e_{i}$ is removed when breaking the $\left(x_{\max }+1\right)$-vertex paths

$$
h_{i}= \begin{cases}1 & e_{i} \in \mathcal{E}_{j} \text { and } e_{i} \notin \mathcal{E}_{j}^{\prime} . \\ 0 & \text { otherwise }\end{cases}
$$

The problem of removing the minimum number of edges from $\mathcal{G}_{j}$ to break all the $\left(x_{\max }+1\right)$-vertex paths can be formulated as

$$
\min \sum_{i=1}^{\left|\mathcal{E}_{j}\right|} h_{i} \quad \text { s.t. } \quad \forall k, \sum_{i=1}^{\left|\mathcal{E}_{j}\right|} h_{i} \cdot g_{i k}>0 .
$$

The constraint ensures each of the $\left(x_{\max }+1\right)$-vertex paths is broken after removing the edges. This is a classic 0-1 integer programming problem and can be nearoptimally solved [14]. 


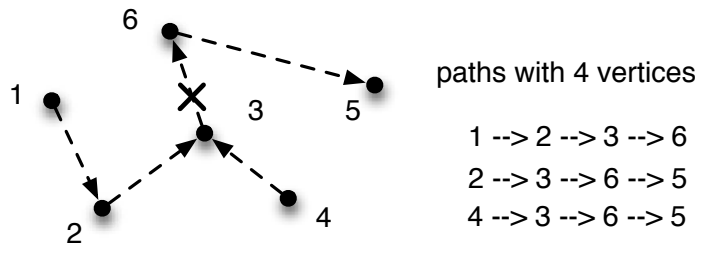

(a)

$$
A=\left|\begin{array}{llllll}
0 & 1 & 0 & 0 & 0 & 0 \\
0 & 0 & 1 & 0 & 0 & 0 \\
0 & 0 & 0 & 0 & 0 & 1 \\
0 & 0 & 1 & 0 & 0 & 0 \\
0 & 0 & 0 & 0 & 0 & 0 \\
0 & 0 & 0 & 0 & 1 & 0
\end{array}\right|
$$

(b)

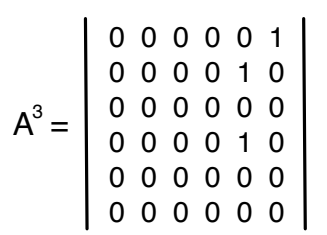

(c)

Figure 7: Demonstration on the identification of cell strings.

\subsubsection{Identifying the Optimal Configuration}

THEOREM 2. The obtained graph $\mathcal{G}_{j}^{\prime}$ after breaking all the $\left(x_{\max }+1\right)$-vertex paths in $\mathcal{G}_{j}$ is acyclic and has no path involving more than $x_{\max }$ vertices.

Proof. This theorem can be proved by contradiction. Let us start with the property that $\mathcal{G}_{j}^{\prime}$ has no path involving more than $x_{\max }$ vertices. If there is a path in $\mathcal{G}_{j}^{\prime}$ involving $x^{\prime}$ vertices and $x^{\prime} \geq x_{\max }+1$, then we can find at least one of its sub-path involving $\left(x_{\max }+1\right)$ vertices. This contradicts with our previous operation which breaks all the $\left(x_{\max }+1\right)$-vertex paths in $\mathcal{G}_{j}$. Next we consider the acyclic property of $\mathcal{G}_{j}^{\prime}$. If $\mathcal{G}_{j}^{\prime}$ is not acyclic, there would be a cycle in $\mathcal{G}_{j}^{\prime}$ involving at least two vertices. Then we can obtain a path involving an arbitrary number of vertices by consecutively traversing along the cycle. Again, this contradicts with the fact that all $\left(x_{\max }+1\right)$-vertex paths have been broken in the previous operation.

For the example shown in Fig. 7 we remove edge $3 \rightarrow$ 6 (which is shared by all the three paths) from $\mathcal{G}_{j}$. The resultant graph $\mathcal{G}_{j}^{\prime}$ has no path involving more than 3 vertices and is acyclic.

As a result, we can apply Hopcroft-Karp algorithm to $\mathcal{G}_{j}^{\prime}$ to obtain a set of feasible disjoint paths based on which the battery charging can be performed, e.g., $\{1 \rightarrow 2 \rightarrow 3\},\{4\}$, and $\{6 \rightarrow 5\}$ in the example shown in Fig. 7. Furthermore, we have the following theorem on the near-optimality of the returned configuration in terms of the number of cell strings.

THEOREM 3. Denote $z$ and $z^{*}$ as the number of paths obtained by our algorithm and those in the optimal solution, respectively. Further denote $u$ as the number of edges removed from $\mathcal{G}_{j}$ when breaking the $\left(x_{\max }+1\right)$ vertex paths (i.e., $\left|\mathcal{V}_{j}\right|-\left|\mathcal{V}_{j}^{\prime}\right|=u$ ), we have

$$
z \leq z^{*}+u \text {. }
$$

This theorem is based on the following observation. For any removed edge, it can at most connect two of these returned paths into one longer path (this connected longer path may not be feasible anymore). Again, let us take the graph shown in Fig. 7 as an example, where edge $3 \rightarrow 6$ is removed from the graph, and the minimum path set obtained by Hopcroft-Karp algorithm is $1 \rightarrow 2 \rightarrow 3$, 4, and $6 \rightarrow 5$. We can see if we add edge $3 \rightarrow 6$ to the three paths, then a longer path $1 \rightarrow 2 \rightarrow 3 \rightarrow 6 \rightarrow 5$ is formed by connecting $1 \rightarrow 2 \rightarrow 3$ and $6 \rightarrow 5$. As a result, if we add all these $u$ removed paths back to $\mathcal{G}_{j}^{\prime}$, the minimum path cover number can be reduced by at most by $u$, and thus the theorem follows.

From theorem 3 we can see that the number of removed edges bounds the gap between our result and the optimal solution. This is also the reason why we want to break all the $\left(x_{\max }+1\right)$-vertex paths by removing the fewest number of edges, as mentioned in Section 5.2.2.

\subsubsection{Amount of Additional Resistors}

After identifying the set of cell strings, we need to determine the amount of resistance (i.e., the number of unit resistors in the associated resistor array) to be connected along each of them to achieve the desired charging current $\hat{I}_{j}$. For a $\mathrm{Path}_{i}$ with $x_{i}$ vertices, we want to add a proper amount of unit resistors to make the achieved charging current $I$ as close as possible to the desired level $\hat{I}_{j}$

$$
\min \left\{\left|I-\hat{I}_{j}\right|\right\} .
$$

By substituting Eq. (10) into (6), we can easily identify the number of added unit resistors by

$$
\arg \min _{y_{i}}\left\{\left|\frac{V-x_{i} \cdot \hat{v}_{c}^{j}}{x_{i} \cdot r+y_{i} \cdot r_{0}}-\hat{I}_{j}\right|\right\} .
$$

\section{EXPERIMENT EVALUATION}

We present our experiment results on the proposed reconfiguration-assisted charging in this section.

\subsection{Experiment Methodology}

In our experiment, we explore the charging performance of a system consisting of 8 NCR18650 Lithiumion battery cells, each with a nominal capacity of 


$$
\left[\begin{array}{llllllll}
0 & 0 & 1 & 0 & 1 & 1 & 0 & 0 \\
1 & 0 & 0 & 1 & 0 & 0 & 1 & 0 \\
0 & 1 & 0 & 0 & 1 & 0 & 0 & 1 \\
1 & 0 & 1 & 0 & 0 & 1 & 0 & 0 \\
1 & 1 & 0 & 0 & 0 & 0 & 0 & 1 \\
0 & 1 & 0 & 0 & 1 & 0 & 1 & 0 \\
1 & 0 & 0 & 1 & 0 & 0 & 0 & 1 \\
0 & 0 & 1 & 0 & 0 & 1 & 1 & 0
\end{array}\right]
$$

Figure 8: Conf. flexibility of the 8-cell system.

$2900 \mathrm{mAh}$. The randomly generated configuration flexibility of the 8-cell system, represented by its adjacent matrix, is shown in Fig. 8 .

We introduce a control parameter $\phi$ to capture the unbalance degree on the voltages of these 8 cells: the initial voltages of these cells are randomly generated from the interval

$$
\left[v_{\text {cutoff }}, v_{\text {cutoff }}+\phi\left(v_{\max }-v_{\text {cutoff }}\right)\right] \quad(\phi \in[0,1]),
$$

where $v_{\text {cutoff }}$ and $v_{\max }$ are set to 3.30 volt and 4.20 volt according to the charging chart of the NCR18650 battery shown in Fig. 1, In this way, a larger $\phi$ indicates higher unbalanced cells.

With a unit resistance of $2 \mathrm{ohm}$ and a 0.002 volt voltage sensor granularity, the entire voltage range $[3.30,4.20]$ during the charging process is divided into 7 intervals: $[3.30,3.517),[3.517,3.723),[3.723,3.956)$, $[3.956,4.193), \quad[4.193,4.195), \quad[4.195,4.197), \quad$ and $[4.197,4.20]$. The first 4 intervals are for the CCChg phase and the latter 3 intervals are for the CV-Chg phase. The 8 cells are then categorized based on their initial voltages. According to the charging chart shown in Fig. 1, the corresponding desired charging currents for cells in these categories are $0.825 \mathrm{~A}, 0.825 \mathrm{~A}$, $0.825 \mathrm{~A}, 0.825 \mathrm{~A}, 0.398 \mathrm{~A}, 0.177 \mathrm{~A}$, and $0.084 \mathrm{~A}$. We then apply the proposed reconfiguration-assisted charging on the battery system to obtain the detailed charging profile for each of these cells. The NEWARE Battery Testing System [9] is adopted to carry out the charging process based on these charging profiles, as shown in Fig. 9.

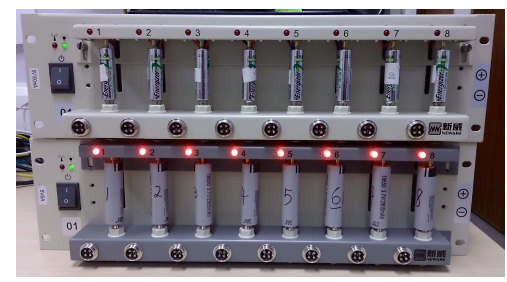

Figure 9: NEWARE Battery Testing System.
As a baseline, we also explore a non-reconfigurable 8-cell system with the configuration of $4 S 2 P$ : two cell strings (i.e., $B_{1} \rightarrow B_{2} \rightarrow B_{3} \rightarrow B_{4}$ and $B_{5} \rightarrow B_{6} \rightarrow$ $\left.B_{7} \rightarrow B_{8}\right)$ are connected in parallel. With this fixed configuration, for system safety, the charging current on each string is determined by the smallest desired charging current of the 4 cells in the string, and the charging process has to be terminated when any of these 4 cells reaches its full voltage (i.e, 4.20 volt) [31,33.

After the charging process finishes, we discharge the cells with a constant current of $0.2 \mathrm{~A}$ till they are discharged to the cutoff voltage 3.30 volt, and the delivered capacities of these 8 cells are recorded as the metric to evaluate the proposed charging algorithm.

\subsection{Experiment Results}

We explore 5 cases with $\phi$ varying from 0.1 to 0.9 , and the initial voltages of individual cells are shown in Table 1 The individual cells delivered capacities with both the proposed charging algorithm and the nonreconfigurable baseline are shown in Table 2, and the overall performance of the 5 explored cases are shown in Fig. 10.

We can see that with the assistance of system reconfiguration, about $2600 \mathrm{mAh}$ capacity can be delivered for each of the cells in all the explored cases. The delivered capacities are stable in terms of both average and standard deviations. Although variance does exist in the delivered capacities, this variance is much smaller when compared with the non-reconfigurable baseline.

We also tested the delivered capacity (with 0.2 A discharge current) after charging the cells by the off-theshelf-charger, similar results (about $2600 \mathrm{mAh}$ ) are obtained as with the proposed charging algorithm. Note that the charge of these cells with the commercial charger is performed individually rather than in-batch as a battery pack.

On the other hand, the delivered capacities show clearly decreasing trend as $\phi$ increases with the nonreconfigurable baseline, e.g., from an average delivered capacity of $2597.2 \mathrm{mAh}$ with $\phi=0.1$ decreased to $2118.4 \mathrm{mAh}$ with $\phi=0.9$, indicating the cells are only charged to about $73 \%$ of their nominal capacity on average. This is caused by two major reasons. First, the charging process has to be terminated when any cell in the string reaches its full capacity for system safety consideration, indicating other cells may not be able to be fully charged. Second, the charging current on a cell string is determined by the smallest desired current of the 4 batteries, and thus reduces the charging efficiency of other cells when different charging currents are desired. These also explain the significant increase of the standard deviation of the delivered capacities as $\phi$ increases. These reasonings are also supported by the fact 
Table 1: Initial Voltages (volt).

\begin{tabular}{|c||c|c|c|c|c|c|c|c|}
\hline Cell & $\mathbf{\# 1}$ & $\mathbf{\# 2}$ & $\mathbf{\# 3}$ & $\mathbf{\# 4}$ & $\mathbf{\# 5}$ & $\mathbf{\# 6}$ & $\mathbf{\# 7}$ & $\mathbf{\# 8}$ \\
\hline \hline$\phi=0.1$ & 3.353 & 3.306 & 3.377 & 3.370 & 3.352 & 3.356 & 3.331 & 3.361 \\
\hline$\phi=0.3$ & 3.473 & 3.439 & 3.456 & 3.372 & 3.394 & 3.437 & 3.533 & 3.336 \\
\hline$\phi=0.5$ & 3.560 & 3.548 & 3.520 & 3.360 & 3.460 & 3.432 & 3.556 & 3.580 \\
\hline$\phi=0.7$ & 3.812 & 3.596 & 3.214 & 3.639 & 3.618 & 3.920 & 3.862 & 3.805 \\
\hline$\phi=0.9$ & 3.710 & 4.044 & 3.960 & 4.072 & 3.862 & 3.428 & 3.995 & 4.058 \\
\hline
\end{tabular}

Table 2: Delivered Capacities (mAh).

\begin{tabular}{|c|c||c|c|c|c|c|c|c|c|}
\hline$\phi$ & Battery & $\mathbf{\# 1}$ & $\mathbf{\# 2}$ & $\mathbf{\# 3}$ & $\mathbf{\# 4}$ & $\mathbf{\# 5}$ & $\mathbf{\# 6}$ & $\mathbf{\# 7}$ & $\mathbf{\# 8}$ \\
\hline \hline \multirow{2}{*}{0.1} & Reconf. & 2619.5 & 2636.8 & 2617.6 & 2670.5 & 2706.3 & 2582.0 & 2657.9 & 2621.9 \\
\cline { 2 - 10 } & Non-Reconf. & 2508.5 & 2607.7 & 2613.7 & 2660.7 & 2611.6 & 2575.6 & 2593.6 & 2606.5 \\
\hline \multirow{2}{*}{0.3} & Reconf. & 2607.6 & 2560.6 & 2608.6 & 2660.0 & 2694.5 & 2567.7 & 2644.4 & 2609.6 \\
\cline { 2 - 10 } & Non-Reconf. & 2597.3 & 627.8 & 2576.8 & 2459.0 & 2432.7 & 2458.2 & 2647.7 & 2276.7 \\
\hline \multirow{2}{*}{0.5} & Reconf. & 2616.4 & 2628.5 & 2611.1 & 2665.2 & 2704.2 & 2577.6 & 2650.8 & 2611.8 \\
\cline { 2 - 9 } & Non-Reconf. & 2622.3 & 2510.3 & 2492.6 & 2304.3 & 2364.4 & 2222.4 & 2526.6 & 2610.6 \\
\hline \multirow{2}{*}{0.7} & Reconf. & 2606.5 & 2614.8 & 2595.6 & 2646.3 & 2682.0 & 2555.1 & 2632.6 & 2593.6 \\
\cline { 2 - 9 } & Non-Reconf. & 2597.9 & 2046.1 & 857.7 & 2253.8 & 1821.6 & 2553.6 & 2499.8 & 2300.0 \\
\hline \multirow{2}{*}{0.9} & Reconf. & 2617.9 & 2633.0 & 2610.9 & 2670.2 & 2714.4 & 2579.4 & 2649.9 & 2606.7 \\
\cline { 2 - 9 } & Non-Reconf. & 1566.4 & 2580.1 & 2340.3 & 2663.3 & 2140.1 & 541.3 & 2501.5 & 2614.3 \\
\hline
\end{tabular}

that in each of the explored cases, the cell with the highest initial voltage in each string (e.g., battery \#1 and \#6 with $\phi=0.7)$ delivers similar capacity as in the reconfiguration-assisted charging process.

\section{SIMULATION EVALUATION}

We have evaluated the performance of the proposed reconfiguration-assisted charging algorithm with smallscale experiment in the previous section. In this section, we further evaluate the system performance through large-scale trace-driven simulations.

\subsection{Simulation Settings}

We simulate a battery system consisting of 20 to 100 NCR18650 cells. The system reconfiguration flexibility, described by the average vertex out-degree $d$ in the abstracted graph representation, varies from 1 to 5 . For a specific cell, its neighbors are randomly selected from other cells in the system. Same as in the experiment section, the cell initial voltages are generated according to (7), and with a unit resistor of $2 \mathrm{ohm}$ and voltage sensor accuracy of 0.002 volt, the entire voltage range $[3.30,4.20]$ is divided into 7 intervals. We also simulate non-reconfigurable battery systems with the configuration of $\frac{N}{4} S 4 P$ as baselines (i.e., 4 parallel connected cell strings each consisting of $\frac{N}{4}$ cells). The desired charging currents and corresponding voltages are obtained from the NCR18650 data sheet as shown in Fig. 1. The results presented are based on a total number of 50 runs.

\subsection{Simulation Results}

Intuitively, the cell unbalance issue becomes more critical when the system scale increases. To verify this, we vary the system scale from 20 to 100 cells with a reconfiguration flexibility of 3 and $\phi=0.5$, and apply the reconfiguration-assisted charging to the system. The cell capacities after the charging processes terminated (with the reconfiguration-assisted charging and the non-reconfigurable system, respectively) are shown in Fig. 11. We can see that in terms of the charged capacity, the reconfiguration-assisted charging achieves stable and competitive performance for all of the explored cases. On the other hand, in the nonreconfigurable system, the charged cell capacities decrease as the system scale increases. This is because the charging process in this case has to be terminated when any of these cells reaches its full voltage, leaving other cells insufficiently charged. This is also supported by the fact that the variance in the charged capacities with the non-reconfigurable system dramatically increases with larger system scales.

As proved in Section [5 the number of cell strings adopted to perform the charging process should be minimized to reduce the energy loss on the additional resistors. To gain more insights on the charging process, we record the number of cell strings when charging the cells in each of the 7 categories with $N=100$ and $\phi=0.8$. Four cases of the system reconfiguration flexibility from 2 to 5 are explored. The average paths number during the charging process are shown 

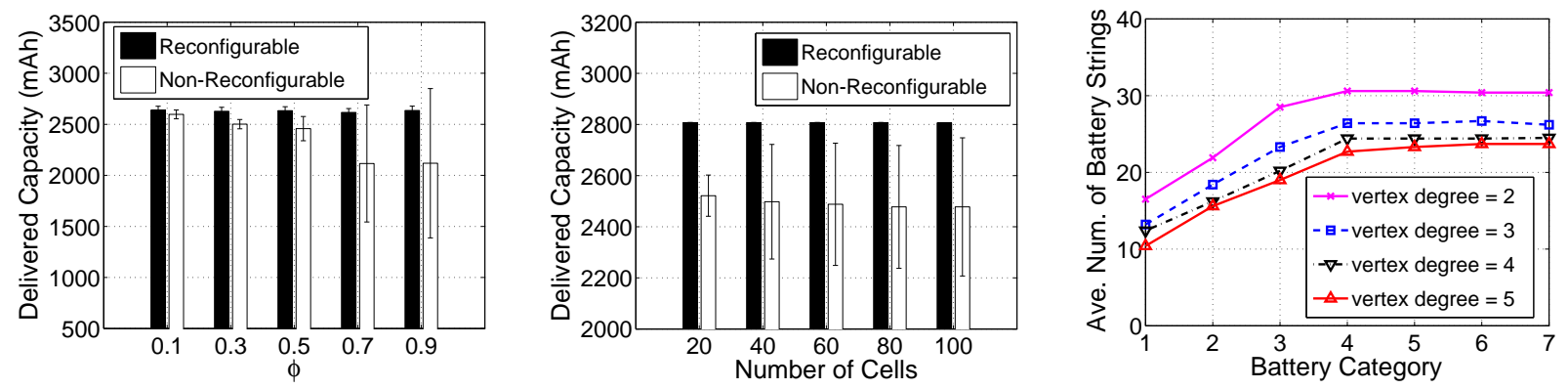

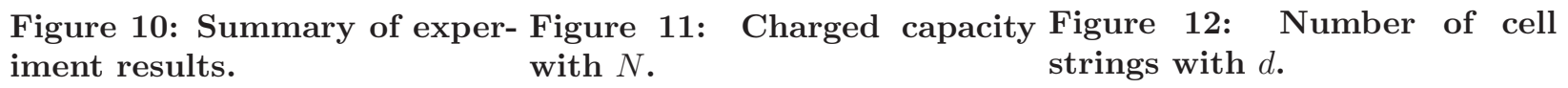

in Fig. 12, We can see the number of cell strings increases as the charging process evolving from the 1st to the 7th category, as more cells need to be considered in this category-by-category charging process. The string number converges to its maximal value when the charging process reaches the 4th category, since all of the cells have to be included into the charging consideration. Specifically, in our simulation, the average numbers of cells involved in the charging of each category are $\{27.9,57.7,91.3,99.8,100,100,100\}$.

Furthermore, a higher reconfiguration flexibility reduces the number of cell strings, which meets our expectations. However, compared with the reduction in the string number when increasing $d$ from 2 to 3 (i.e., from about 30.4 to 26.2 strings), further increasing $d$ from 4 to 5 has a much smaller effect in reducing the number of strings (i.e., from about 24.5 to 23.7 strings), indicating the effect of the reconfiguration flexibility in reducing the number of cell strings approaches its upper bound. This observation is of significant practical value because a higher reconfiguration flexibility also imposes higher system implementation complexity and cost.

\section{RELATED WORK}

Large-scale battery systems are commonly adopted in practice [1,3,40. However, besides providing powerful energy supply, the large-scale battery systems also make the cell unbalance issue more critical 21] and thus makes the design of efficient battery management system challenging [15, 38, 41].

Exploring the system reconfiguration flexibility is a new dimension to improve the large-scale battery system, and has attracted a lot of research attentions and funding opportunities 2, 11. Additional supplementary components such as switches and relays are needed to make the battery system reconfigurable, and many works investigating how to offer the maximal reconfiguration flexibility with the fewest supplementary components have been reported [13, 28, 31. In large-scale battery systems, it may not be desirable nor feasible for all the cells to be discharged in the same manner, and thus many works on the discharge management of the cells in the system have been reported [16, 17, 36, 44. The discharge management is especially important to achieve a high system energy efficiency when considering the recovery 32,45] and rate-capacity 24] effects of batteries. The real-time system states are needed for the discharge management to be applied. However, the system monitoring also introduces additional energy cost and system complexity. Kim et al. have explored how to effectively achieve the real-time system monitoring in 34. Jin et al. have also explored how to improve the reliability of the battery system based on the reconfiguration flexibility 28.

However, to the best of our knowledge, no work on exploring how to utilize the reconfiguration flexibility to improve the charging process of the battery system has been reported yet, and our work is the first attempt to demonstrate how the system reconfiguration flexibility can assist when charging the system. Note that although the charging schedule of cells has been thoroughly investigated in 32, this schedule is only based on the cell states and does not consider the offered system reconfiguration flexibility directly.

\section{CONCLUSIONS}

In this paper, we have demonstrated the effectiveness of utilizing the system reconfiguration flexibility to achieve a high-efficient charging process for largescale Lithium-ion battery systems. By categorizing the cells according to their voltages, the reconfigurationassisted charging process is evolutionarily carried out in a category-by-category manner. A graph-based algorithm has been designed to identify the desired system configuration to charge cells in a given category. The performance of the reconfiguration-assisted charging has been verified through both small-scale experiment and large-scale trace-driven simulations. Our future work focus on prototyping with moderate scales. 
Acknowledgment: This research was supported in part by iTrust IGDSi1301013, SUTDZJU/RES/03/2011, CNS-0845994, and NSFC grant No. 61303202 .

\section{REFERENCES} [1] Aircraft Electrical Systems.
http://www.pilotfriend.com/training/flight_training/tech/etecthtom In IEEE RTSS'13@Work, 2013.
[2] ARPA-E.

[2] ARPA-E. http://www .greencarcongress.com/2012/08/arpae-20120802.hthaximum matchings in bipartite graphs. SIAM

[3] The Basics of Backup Power. http://www.smps.us/backuppower.html

[4] Battery Charging Tutorial. http://www . chargingchargers . com/tutorials/charging.html

[5] Boeing 787 Dreamliner battery problems. http://en.wikipedia.org/wiki/Boeing_787_Dreamliner_batter_yejpletbledms

[6] Elithion Battery Pack. http://elithion.com/battery_packs.php

[7] Lithium-ion Rechargeable Batteries Technical Handbook. http://www. sony.com.cn/products/ed/battery/download.p

[8] LM2576, LM3420, LP2951, LP2952. http://www.ti.com/lit/an/snva557/snva557.pdf

[9] Neware Battery Testing System. http://www. batteryspace.com/prod-specs/V-BTS8-MA.pdf

[10] Panasonic NCR18650 Li-ion Battery.

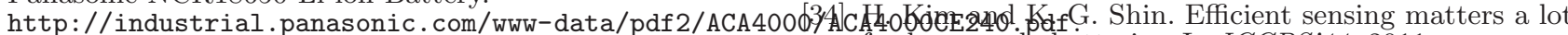

11] Reconfigurable Battery Packs.

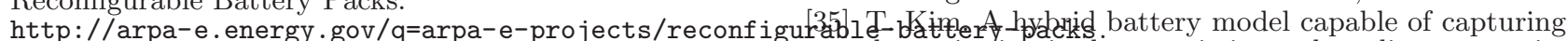

[12] Serial and Parallel Battery Configurations.

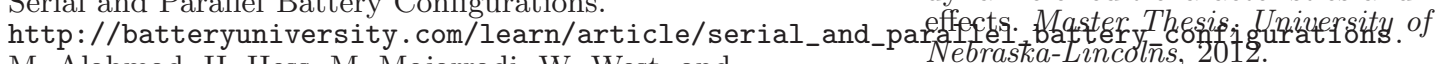

[13] M. Alahmad, H. Hess, M. Mojarradi, W. West, and J. Whitacre. Battery switch array system with application for JPL's rechargeable micro-scale batteries. Journal of Power Sources, 177(2):566 - 578, 2008.

[14] S. Boyd and L. Vandenberghe. Convex Optimization. Cambridge University Press, 2004.

[15] C. F. Chiasserini and R. Rao. Energy efficient battery management. IEEE Journal on Selected Areas in Communications, 19(7):1235-1245, 2001.

[16] S. Ci, J. Zhang, H. Sharif, and M. Alahmad. Dynamic reconfigurable multi-cell battery: A novel approach to improve battery performance. In APEC'12, 2012.

[17] S. Ci, J. Zhang, H. Sharif, and M. Alahmadu. A novel design of adaptive reconfigurable multiple battery for power-aware embedded networked sensing systems. In GLOBECOM'12, 2012.

[18] T. H. Cormen, C. E. Leiserson, R. L. Rivest, and C. Stein. Introduction to Algorithms. MIT Press, 2001.

[19] S. Diaz, H. Jain, Y. Pant, W. Price, and R. Mangharam. Protodrive: An experimental platform for electric vehicle energy scheduling and control. In IEEE RTSS'12@Work, 2012.

[20] L. R. Ford and D. R. Fulkerson. Flows in networks. Princeton University Press, 1962.

[21] R. Garcia-Valle and J. P. L. (eds.). Electric vehicle integration into modern power networks. Power Electronics and Power Systems, 2013.

[22] Y. Gu, L. He, T. Zhu, and T. He. Achieving energy synchronized communication in energy-harvesting wireless sensor networks. ACM Transactions on Embedded Computing Systems, 2014.

[23] L. He, P. Cheng, Y. Gu, J. Pan, T. Zhu, and C. Liu. Mobile-to-mobile energy replenishment in mission-critical robotic sensor networks. In IEEE INFOCOM'14, 2014.

[24] L. He, L. Gu, L. Kong, Y. Gu, C. Liu, and T. He.
Exploring adaptive reconfiguration to optimize energy efficiency in large-scale battery systems. In $R T S S^{\prime} 13$, 2013.

[25] L. He, Y. Gu, J. Pan, and T. Zhu. On-demand charging in wireless sensor networks: Theories and applications. In IEEE MASS'13, 2013.

26] L. He, S. Ying, and Y. Gu. Reconfiguration-based energy optimization in battery systems: a testbed Journal on Computing, 2(4):225-231, 1973.

[28] F. Jin and K. G. Shin. Pack sizing and reconfiguration for management of large-scale batteries. In ICCPS'12, 2012.

H. Kiehne. Battery Technology Handbook. Marcel

उ० E. Kim, J. Lee, and K. G. Shin. Real-time prediction of battery power requirements for electric vehicles. In ICCPS'13, 2013.

[31] H. Kim and K. G. Shin. On dynamic reconfiguration of a large-scale battery system. In RTAS'09, 2009.

[32] H. Kim and K. G. Shin. Scheduling of battery charge, discharge, and rest. In RTSS'09, 2009.

[33] H. Kim and K. G. Shin. Dependable, efficient, scalable architecture for management of large-scale batteries. In $I C C P S^{\prime} 10,2010$.

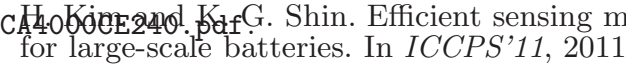

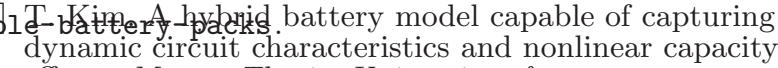

[36] T. Kim, W. Qiao, and L. Qu. Series-connected self-reconfigurable multicell battery. In APEC'11, 2011.

[37] T. Kim, W. Qiao, and L. Qu. A series-connected self-reconfigurable multicell battery capable of safe and effective charging/discharging and balancing operations. In APEC'12, 2012.

[38] D. Lim and A. Anbuky. A distributed industrial battery management network. ACM Trans. on Indus. Elec., 51(6):1181-1193, 2004.

[39] D. Linden and T. B. Reddy. Handbook of Baterries (3rd ed.). McGraw-Hill, 2001.

[40] J. Markoff. Pursuing a battery so electric vehicles can go the extra miles. http://www . nytimes.com/2009/09/15/science/15batt.html

[41] D. Rakhmatov and S. Vrudhula. Energy management for battery-powered embedded systems. $A C M$ Transactions on Embedded Computing Systems, 2:277-324, 2003.

[42] D. Reinhard. Graph Theory (3rd ed.). Springer, 2005.

[43] S.C.Ntafos and S. L. Hakimi. On path cover problems in digraphs and applications to program testing. IEEE Transactions on Software Engineering, 5(5):520-529, 1979.

[44] H. Visairo and P. Kumar. A reconfigurable battery pack for improving power conversion efficiency in portable devices. In ICCDCS'08, 2008.

[45] F. Zhang and Z. Shi. Optimal and adaptive battery discharge strategies for cyber-physical systems. In IEEE CDC'09, 2009. 